(C) Elsevier Sequoia S.A., Lausanne - Printed in the Netherlands

\title{
ONTOGENETIC DETERMINANTS OF BEHAVIORAL RESPONSES TO MORPHINE IN RATS
}

\author{
RICHARD J. KATZ
}

Mental Health Research Institute, Department of Psychiatry, University of Michigan Medical Center, Ann Arbor, MI 48109 (U.S.A.)

(Received February 15, 1979)

\section{Summary}

Male Sprague-Dawley rats ranging in age from 20 to 110 days were injected with two doses of morphine sulphate, and examined for behavioral activation and catalepsy. Behavioral activation increased with age, while sensitivity to catalepsy declined. Ontogenetic and pharmacological responses suggest possible separate mediation of activation and catalepsy.

Psychoactive drugs are known to have distinctive and often disparate behavioral effects when given to animals of different ages. Such ontogenetic differences are most readily seen with compounds affecting aminergic systems, although these effects are not unique to the latter [1]. Opiates, particularly at low doses are known to increase behavioral activity in rats, while higher doses produce catalepsy. Only limited work has addressed potential age-related differences in function. Such an examination would be valuable in further characterizing activation and catalepsy mediating systems, and their interrelations. Moreover, given the established abuse potential of morphine and related opiates, a study of the differential effects of the former in young organisms might at least indirectly point to potentially unique problems of dependence. Accordingly, we have tested rats at various ages and at two behaviorally distinctive doses of drug. Our findings suggest clear ontogenetic effects upon behavioral arousal and catalepsy.

\section{Methods of procedure}

\section{Subjects}

Male Sprague-Dawley rats ranging in age from 20 to 110 days and in weight from 35 - $380 \mathrm{~g}$ were obtained locally (Charles River Farms, Portage, MI) and group housed six per cage in standard $16.5 \times 30.5 \times 30.5 \mathrm{~cm}$ stainless-steel cages. Group housing was employed because of the established inhibitory effects of isolation upon morphine activation [2] . Food (Teklad 
$4.0 \%$ fat diet S-0836) and tap water were continuously available, and $12 \mathrm{~h}$ lighting cycles were automatically programmed (lights on $=8.00-20.00 \mathrm{~h}$ ). Because the youngest group of subjects was not weaned, it was housed with the natural mother.

\section{Apparatus}

Subjects were individually tested on commercially available field-sensitive activity monitors (Stoelting, Chicago, IL). Four platforms were calibrated to within $5 \%$ of each other and set to register gross body movement. Polypropylene containers $48 \times 27 \times 20 \mathrm{~cm}$ (Scientific Products Series 140) were placed upon the monitors with fresh pine-chip bedding. Assignment of platforms to treatment groups was systematically rotated during the series of tests. A continuous background of $20-30 \mathrm{~dB}$ masking noise was provided by an air circulation system.

\section{Behavioral procedure}

All testing was carried out between $15-18 \mathrm{~h}$ (i.e. in the middle of the lights-on period). Subjects were individually placed in the testing apparatus and allowed to habituate for approximately $50 \mathrm{~min}$. Rats were then briefly removed and injected with drug or vehicle, and immediately replaced for behavioral observation and testing. Motor activity was automatically recorded by remote sensors. Behavioral assessment of catalepsy, a second opiate-mediated syndrome, was based upon a simple presence/absence measure. If animals remained immobile in their cages showing odd prolonged $(>30 \mathrm{sec})$ limb placement, they were rated as cataleptic. Ten-minute recording intervals were used throughout and sessions lasted $100 \mathrm{~min}$. Catalepsy was rated between 15 - 45 min for all animals.

\section{Statistical design}

Eighteen rats were tested for each of four ages $(20,50,80$, and 110 days). A three-group design was used. Six subjects each were tested with vehicle or one of two drug doses. All analysis is presented as mean and standard error. Due to large differences in variance across conditions, all analyses were based upon Friedman's two-way analysis of variance, and Fisher's exact test [3].

\section{Drugs}

A relatively low $(1.5 \mathrm{mg} / \mathrm{kg})$ dose of morphine was used to produce behavioral activation. The same dose and one higher dose $(5.0 \mathrm{mg} / \mathrm{kg})$ were used in the examination of catalepsy. All drugs were administered intraperitoneally in a $0.9 \%$ sodium chloride vehicle solution. Typically $1 \mathrm{ml} / \mathrm{kg}$ injection volumes were employed; however, to assure accuracy, all animals weighing less than $100 \mathrm{~g}$ were injected with $10 \mathrm{ml} / \mathrm{kg}$. Doses were based upon pilot data in our laboratory and previously published reports $[2,4,5]$. 
Results

Effects of drug vs. vehicle upon activity

For all groups, injection of a low dose of opiate produced some degree of behavioral activation in comparison with control conditions $\left(\chi_{r}^{2}=10,6\right.$ 10,10 for the four age groups, $p<0.01,0.02,0.01,0.01$, respectively). In addition, a clear age-related effect of drug efficacy was apparent across the four drug conditions. Morphine-induced activity increased with age, to adulthood, with a slight relative decline at the final 110 day test $\left(\chi_{r}^{2}=28\right.$, d.f. $=3, p<0.01$ ). All results are presented graphically in Fig. 1 . To verify this effect further, we examined maximal behavioral activation after drugs, irrespective of time. Median values of peak activity across the four groups were $10,199,472$, and 247 . To facilitate comparison across conditions, Table 1 offers additional summary measures of activation scores.
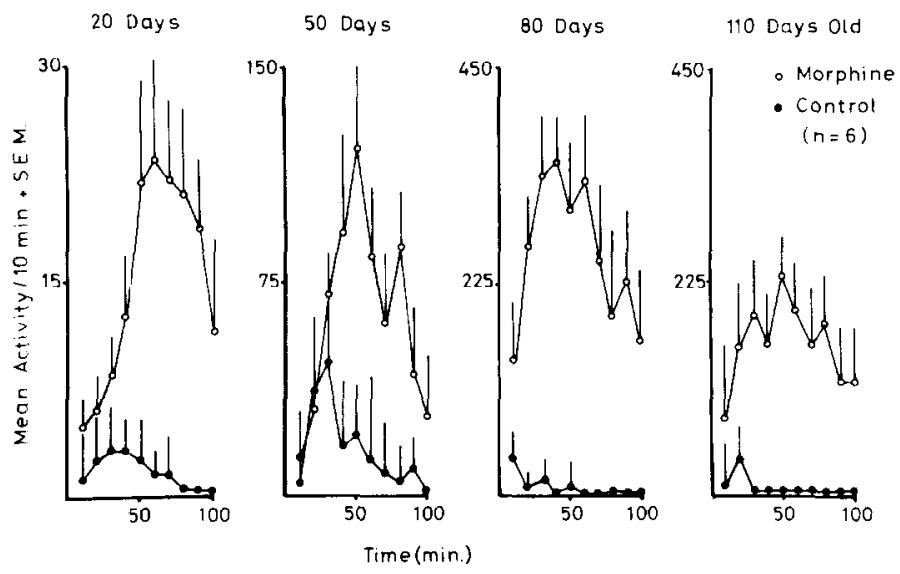

Fig. 1. Morphine-induced behavioral activation in rats of different ages. Mean + s.e.m. Solid line is morphine $(1.5 \mathrm{mg} / \mathrm{kg})$ injected. Broken line is vehicle injected. Note that ordinate is changed across ages to maximize individual behavioral descriptions.

TABLE 1

Effect of morphine on activity: age-related differences (mean values per $100 \mathrm{~min}$ )

\begin{tabular}{lrrrr}
\hline & \multicolumn{2}{c}{ Age (days) } & & \\
\cline { 2 - 5 } & 20 & 50 & 80 & 110 \\
\hline Control & 6 & 272 & 150 & 90 \\
Experimental & 125 & 511 & 2080 & 1250 \\
\hline
\end{tabular}

Summary measures represent the same subjects as in Fig. 1. 


\section{Catalepsy}

An examination of the lower dose of morphine (Fig. 2) indicated a significant decline in catalepsy with age $(p<0.02$ comparing days 20 and 110 by Fisher exact test). The higher dose produced catalepsy at all ages although again a slight but non-significant decline was present with increasing age ( $p>0.05$ by Fisher exact test on extreme age values).

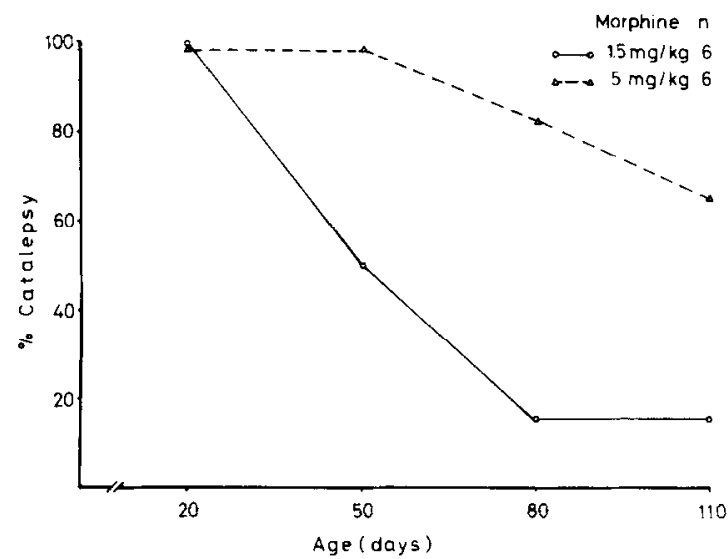

Fig. 2. Percentage catalepsy in rats of different ages after two doses of morphine. See text for a description of behavioral procedures.

\section{Discussion}

In the present experiment low doses of an opiate produced two agerelated effects. Morphine caused behavioral activation which increased with age, and catalepsy which decreased with age. Given the relatively small size of the youngest group of rats, it might be questioned if the monitors were capable of detecting their motor activity. It should be noted that we have tested mice, animals of even smaller weight, using identical procedures and monitor sensitivity, and have found activation to opiates equivalent to that seen here with older animals [6]. The observed effect is therefore probably not a function of apparatus sensitivity.

Since a single dose of opiate produced two oppositely signed effects over time this may indicate differential mediation of these two behaviorally distinctive syndromes. Higher doses of opiate continued to elicit catalepsy throughout testing and this may suggest that the system mediating the latter is functional even in young animals and relatively less subject to developmental effects. The activation-mediating system, on the other hand, may represent an age-sensitive psychobiological marker of opiate effects.

The precise central mediation of the observed behavioral effects remains to be determined. It might be speculated that the maturational differences in behavior reflect perhaps a concomitant change in the availability of some mediating transmitter, or perhaps altered receptor number or sensitivity. 
Whatever the cause of these effects, however, they suggest that opiate systems, or systems associated with the latter, show maturational changes which last up to the adulthood of the rat.

In conclusion, the present studies demonstrate age-dependent behavioral sensitivity to opiates, and this may imply a functional dissociation of the underlying systems. These findings support heterogeneous roles for opiate systems, and may point to developmental factors which modify sensitivity to opiates.

\section{Acknowledgements}

Support was in part provided by postdoctoral grant MH 07417 from the National Institute of Mental Health, through the Mental Health Research Institute. Dr. Barbara B. Turner donated the animals used in this study. The editorial assistance of Mary Sies and Elizabeth Romkema is gratefully acknowledged. I am grateful to Dr. Huda Akil for the advice she offered on an earlier draft of the manuscript.

\section{References}

1 P. D. Mabry and B. A. Campbell, Developmental psychopharmacology, in L. L. Iversen, S. D. Iversen and S. H. Snyder (eds.), Handbook of Psychopharmacology, vol. 7, Principles of Behavioral Pharmacology, Plenum, New York, 1978, pp. 393 - 444.

2 D. M. Katz and $H$. Steinberg, Long term isolation in rats reduces morphine response. Nature, 228 (1970) $469-471$.

3 S. Siegel, Non-Parametric Statistics for the Behavioral Sciences. McGraw-Hill, New York, 1956.

$4 \mathrm{~K}$. Kuschinsky and $\mathrm{O}$. Hornykiewicz, Morphine catalepsy in the rat: Relation to striatal dopamine metabolism. Eur. J. Pharmacol., 19 (1972) 119 - 122.

$5 \mathrm{~S}$. Norton, The structure of behavior of rats during morphine induced hyperactivity. Commun. Psychopharmacol., 1 (1977) 333 - 341.

6 R. J. Katz, B. J. Carroll and G. Baldrighi, Behavioral activation by enkephalins in mice. Pharmacol. Biochem. Behav., 8 (1978) 493 - 496. 